\title{
On the Conjecture of Birch and Swinnerton-Dyer for an Elliptic Curve of Rank 3
}

\author{
By Joe P. Buhler, Benedict H. Gross and Don B. Zagier
}

\begin{abstract}
The elliptic curve $y^{2}=4 x^{3}-28 x+25$ has rank 3 over Q. Assuming the WeilTaniyama conjecture for this curve, we show that its $L$-series $L(s)$ has a triple zero at $s=1$ and compute $\lim _{s \rightarrow 1} L(s) /(s-1)^{3}$ to 28 decimal places; its value agrees with the product of the regulator and real period, in accordance with the Birch-Swinnerton-Dyer conjecture if III is trivial.
\end{abstract}

The object of this note is to verify the conjecture of Birch and Swinnerton-Dyer numerically (to high accuracy) for the elliptic curve

$$
E: y^{2}=4 x^{3}-28 x+25 \text {. }
$$

The conductor of $E$ is 5077 , which is apparently the smallest conductor for a curve of rank 3 over $\mathbf{Q}$. Since previous accurate numerical verifications were done for modular curves of rank 0 or 1 , and these can now be confirmed theoretically [2], [4], it seemed desirable to test the conjecture for a curve of larger rank.

We assume some familiarity with the theory of elliptic curves; good references are [3] and [5].

1. The Canonical Height Function. One of the main ingredients in the BirchSwinnerton-Dyer formula is the regulator, i.e., the determinant of the matrix expressing the canonical height pairing on $E(\mathbf{Q}) \otimes \mathbf{R}$ with respect to a $\mathbf{Z}$-basis of $E(\mathbf{Q}) / E(\mathbf{Q})_{\text {tors }}$. In this section we describe how to calculate the canonical height of a point $P \in E(\mathbf{Q})$.

We first recall the definition. The global minimal model for $E$ has the form

$$
y^{2}+y=x^{3}-7 x+6
$$

obtained by replacing $y$ by $2 y+1$ in (1) and dividing by 4 ; this equation has discriminant $\Delta=5077$. If $P \in E(\mathbf{Q})$, then the naive height of $P$ is defined as

$$
h(P)=\log \max (|a|, b), \quad x(P)=a / b, b>0,(a, b)=1
$$

(here it does not matter whether we use model (1) or (2) for $E$, as the $x$-coordinates are the same); the canonical height is the unique quadratic form $\hat{h}$ on $E(\mathbf{Q}) \otimes \mathbf{R}$ such that $\hat{h}(P)-h(P)$ is bounded, and the canonical height pairing is the associated bilinear form $\left\langle P, P^{\prime}\right\rangle=\frac{1}{2}\left(\hat{h}\left(P+P^{\prime}\right)-\hat{h}(P)-\hat{h}\left(P^{\prime}\right)\right)$. The definition of $\hat{h}$ immediately implies the formula $\hat{h}(P)=\lim _{n \rightarrow \infty} n^{-2} h(n P)$, but this is not convenient for calculations. A formula which is usable is

$$
\hat{h}(P)=\log b+F(x(P)) \text {, }
$$

Received March 20, 1984; revised June 11, 1984.

1980 Mathematics Subject Classification. Primary 14K07, 14G10.

1985 American Mathematical Society $0025-5718 / 85 \$ 1.00+\$ .25$ per page 
where $b$ denotes the denominator of $x(P)$ as in (3) and $F(x)$ is the real-valued function defined by

$$
\begin{gathered}
F(x)=\log |x|+\sum_{n=0}^{\infty} 4^{-n-1} \log z_{n}, \\
z_{n}=1+\frac{14}{x_{n}^{2}}-\frac{50}{x_{n}^{3}}+\frac{49}{x_{n}^{4}}, \quad x_{0}=x, x_{n+1}=\frac{x_{n}^{4}+14 x_{n}^{2}-50 x_{n}+49}{4 x_{n}^{3}-28 x_{n}+25} .
\end{gathered}
$$

Near $x=0$ the first two terms in (5) become infinite, but we can combine them to obtain

$$
F(x)=\frac{1}{4} \log \left(x^{4}+14 x^{2}-50 x+49\right)+\sum_{n=1}^{\infty} 4^{-n-1} \log z_{n},
$$

a formula which now makes sense for all $x$. Note that the formula relating $x_{n+1}$ to $x_{n}$ is the formula relating $x(2 P)$ to $x(P)$ for $P \in E$, so that $x_{n}=x\left(2^{n} P\right)$. In particular, $x_{n} \geqq e_{3}=1.946 \ldots$ for $n \geqq 1$, where $e_{1}<e_{2}<e_{3}$ denote the roots of the polynomial $4 x^{3}-28 x+25$, so $z_{n}$ lies between 1 and $1.328 \ldots$ and $\log z_{n}$ between 0 and $0.284 \ldots$ Therefore the series in (5) or (6) converges very rapidly and we can calculate $\hat{h}(P)$ to any desired degree of accuracy.

Formula (4) is the specialization to our case of a general recipe of Tate [6] for computing heights; indeed, $F(x(P))$ is Tate's formula for the infinite component of $\langle P, P\rangle$ while ord $(b) \log p$ ( $p$ prime) gives the $p$-component of the canonical height (even for the prime $p=5077$ of bad reduction, since the fiber of the Néron model at $p$ is irreducible). However, Tate's result, although quoted in the literature, has not yet been published, so we give a direct proof of (4) in our case. By virtue of the definition, it will suffice to show that the expression on the right-hand side of (4) differs by a bounded amount from $h(P)$ and is multiplied by 4 if $P$ is replaced by $2 P$. By the formula already cited, replacing $P$ by $2 P$ replaces $x(P)=a / b$ by $x(2 P)=a^{*} / b^{*}$, where

$$
a^{*}=a^{4}+14 a^{2} b^{2}-50 a b^{3}+49 b^{4}, \quad b^{*}=4 a^{3} b-28 a b^{3}+25 b^{4} .
$$

We claim that $b^{*}$ is the exact denominator of $x(2 P)$. Indeed, an elementary calculation with g.c.d.'s shows that $\left(a^{*}, b^{*}\right)=1$ for any integers $a, b$ with $(a, b)=1$ unless $a \equiv 92 b(\bmod 5077)$, in which case $5077 \mid\left(a^{*}, b^{*}\right)$. But this cannot happen here, since $4 x^{3}-28 x+25=4(x-92)^{2}(x+184)+5077(20 x-1227)$ would be divisible by 5077 but not by $5077^{2}$ if $x$ were $\equiv 92(\bmod 5077)$ and hence, could not be a square. (This is an elementary restatement of the fact that the Néron model at 5077 has only one component.) On the other hand, replacing $P$ by $2 P$ replaces $x_{n}, z_{n}$ by $x_{n+1}, z_{n+1}$ in (5), so

$$
\begin{aligned}
F(x(2 P)) & =\log |x(2 P)|+\sum_{n=0}^{\infty} 4^{-n-1} \log z_{n+1} \\
& =\log |x(2 P)|+4\left(F(x)-\log |x|-4^{-1} \log z_{0}\right) \quad(x=x(P)) \\
& =4 F(x)-\log \left(4 x^{3}-28 x+25\right) \\
& =4(F(x(P))+\log b)-\log b^{*}
\end{aligned}
$$


proving the second assertion. As to the difference of $h$ and $\hat{h}$, we can write (3) as $h(P)=\log b+\log \max (|a| / b, 1)$, so

$$
\hat{h}(P)-h(P)=F(x)-\log \max (|x|, 1) \quad(x=x(P)) .
$$

If $x \geqq e_{3}=1.94 \ldots$ is in the right-hand component of $E(\mathbf{R})$, then the same is true for all $x_{n}(n \geqq 0)$, so $1 \leqq z_{n} \leqq 1.328 \ldots$ for all $n$ in (5) and therefore

$$
0 \leqq F(x)-\log x \leqq \sum_{n=0}^{\infty} 4^{-n-1} \log (1.328 \ldots)=0.0947 \ldots
$$

The other component $e_{1} \leqq x \leqq e_{2}$ of $E(\mathbf{R})$ is compact and we easily find the minimum and maximum of $F(x)-\log \max (|x|, 1)$ there to be $0.4006 \ldots$ and $1.205 \ldots$ (obtained for $x=e_{1}$ and $x=-1$, respectively; see Figure 1). Hence in all cases we have

$$
h(P) \leqq \hat{h}(P) \leqq h(P)+1.205 \ldots
$$

This completes the proof of (4). We remark that the difference between the naive and canonical heights on elliptic curves has been studied by several authors (cf. [7] and the literature cited there) but that the inequality (7) is much sharper than the one obtained by specializing their results, suggesting that some improvements in the general case may still be possible.

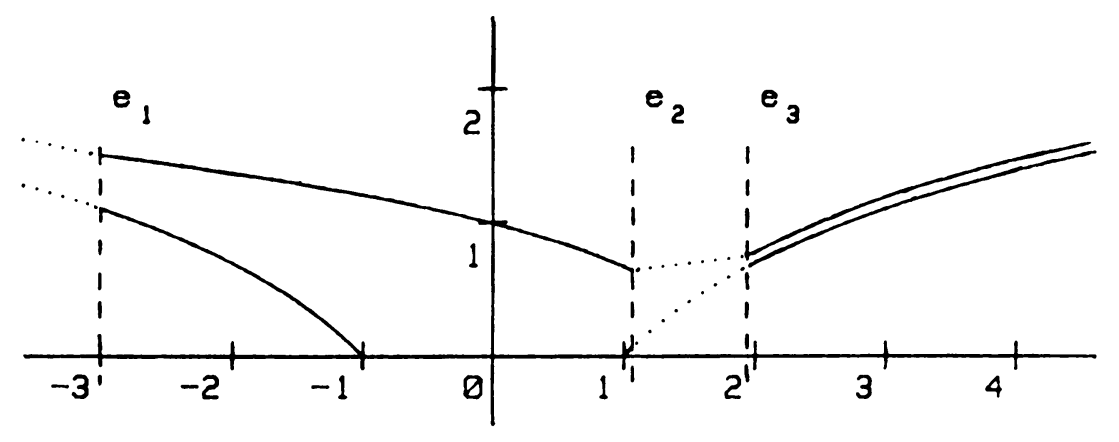

Figure 1

The functions $F(x)$ and $\log \max (|x|, 1)$

2. The Mordell-Weil Group and the Regulator. Let $N_{p}(p \neq 5077)$ denote the cardinality of $E(\mathbf{Z} / p \mathbf{Z})$, i.e., 1 plus the number of solutions of (2) in integers modulo $p$. Then $\left|E(\mathbf{Q})_{\text {tors }}\right|$ must divide $N_{p}$ for all $p>2$; since $N_{3}=7$ and $N_{5}=10$ it follows that $E(\mathbf{Q})$ is free Abelian. We claim that it is of rank 3, generated by the three points

$$
P_{0}=(0,2), \quad P_{1}=(1,0), \quad P_{2}=(2,0)
$$

It follows from Eq. (7) that these are the only points (up to sign) with canonical height less than 1 , since $h(P) \leqq \hat{h}(P) \leqq 1$ implies (cf. (3)) $\max (|a|, b) \leqq e$ and hence 
(since $b$ is always a square) $b=1, a \in\{-2,-1,0,1,2\}$; of these five candidates, only $a=0,1,2$ lead to points with $\hat{h}(P)<1$. On the other hand, one sees by a 2-descent (cf. [1]) that $P_{0}, P_{1}, P_{2}$ generate $E(\mathbf{Q}) / 2 E(\mathbf{Q})$, which is of rank 3 over $\mathbf{Z} / 2 \mathbf{Z}$. These two facts and the fact that $E(\mathbf{Q})$ is torsion-free imply by the usual proof of the Mordell-Weil theorem (cf. any text on elliptic curves) that $E(\mathbf{Q})=\mathbf{Z} P_{0}+\mathbf{Z} P_{1}+\mathbf{Z} P_{2}$ as claimed. Using the algorithm of Section 1 we can calculate the entries of the matrix

$$
A=\left(\left\langle P_{i}, P_{j}\right\rangle\right)_{0 \leqq i, j \leqq 2}=\left(\begin{array}{rrr}
.9909 \ldots & -.2365 \ldots & -.2764 \ldots \\
-.2365 \ldots & .6682 \ldots & .0333 \ldots \\
-.2764 \ldots & .0333 \ldots & .7670 \ldots
\end{array}\right)
$$

to any desired accuracy. The regulator is the determinant of this matrix:

$$
R=\operatorname{det} A=.417143558758383969817119544618093 \ldots
$$

As an illustration, we have given the representations of $P$ as $n_{0} P_{0}+n_{1} P_{1}+n_{2} P_{2}$ and the naive and canonical heights of $P$ for 18 integral points $P \in E(\mathbf{Q})$ in Table 1; the canonical heights can be computed either by the algorithm of Section 1 or as $\left(n_{0} n_{1} n_{2}\right) A\left(n_{0} n_{1} n_{2}\right)^{t}$. One has of course also the negatives $-P=(x,-y-1)=$ $-n_{0} P_{0}-n_{1} P_{1}-n_{2} P_{2}$ with the same heights. The large number of 36 integral points seems to be typical of curves with a high rank relative to their conductor.

TABLE 1

Integral points on $E$

\begin{tabular}{rrrrrrr}
\hline \multicolumn{1}{c}{$x$} & $y$ & $n_{0}$ & $n_{1}$ & $n_{2}$ & $\hat{h}(P)$ & \multicolumn{1}{c}{$h(P)$} \\
\hline-3 & 0 & 0 & -1 & -1 & 1.50192454 & 1.09861229 \\
-2 & 3 & 0 & -1 & 1 & 1.36857251 & .69314718 \\
-1 & 3 & -1 & 0 & -1 & 1.20508110 & 0.00000000 \\
0 & 2 & 1 & 0 & 0 & .99090633 & 0.00000000 \\
1 & 0 & 0 & 1 & 0 & .66820517 & 0.00000000 \\
2 & 0 & 0 & 0 & 1 & .76704336 & .69314718 \\
3 & 3 & 1 & 1 & 0 & 1.18592770 & 1.09861229 \\
4 & 6 & -1 & -1 & -1 & 1.46677848 & 1.38629436 \\
8 & 21 & 1 & -1 & 0 & 2.13229530 & 2.07944154 \\
11 & 35 & -1 & -1 & 1 & 2.43916362 & 2.39789527 \\
14 & 51 & 0 & 2 & 0 & 2.67282066 & 2.63905733 \\
21 & 95 & 0 & 0 & -2 & 3.06817342 & 3.04452244 \\
37 & 224 & -2 & 0 & -1 & 3.62493152 & 3.61091791 \\
52 & 374 & 1 & -1 & 2 & 3.96137952 & 3.95124372 \\
93 & 896 & 2 & 2 & 1 & 4.53836901 & 4.53259949 \\
342 & 6324 & -2 & 0 & 1 & 5.83640586 & 5.83481074 \\
406 & 8180 & 0 & 2 & 2 & 6.00769815 & 6.00635316 \\
816 & 23309 & 1 & 3 & -1 & 6.70508531 & 6.70441435 \\
\hline
\end{tabular}




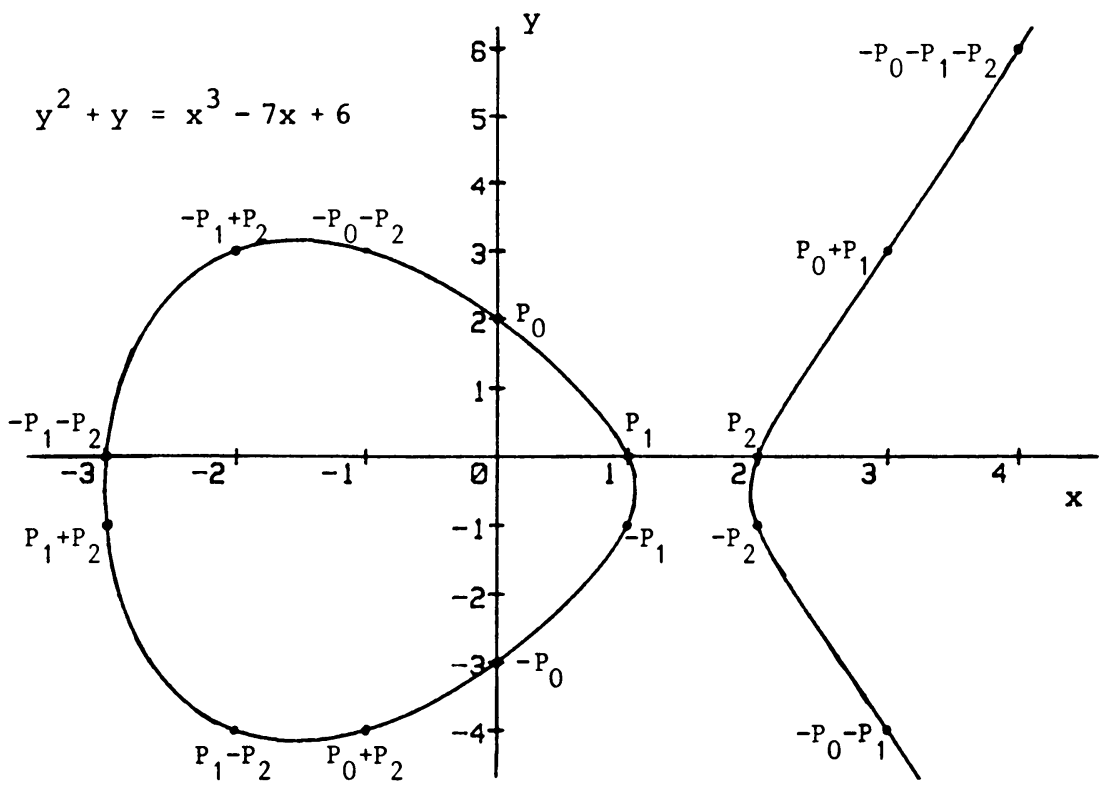

FIGURE 2

Integral points on $E$

3. The Real Period. The group $E(\mathbf{R})$ has two connected components. Let $\omega=$ $d x /(2 y+1)$ be a Néron differential on $E$ over $\mathbf{Z}$, and $|\omega|$ the associated measure on $E(\mathbf{R})$. The real period $\Omega$ is defined by

$$
\Omega=\int_{E(\mathbf{R})}|\omega|=2 \int_{E(\mathbf{R})^{0}}|\omega|
$$

If we write $(0.1)$ in the form $y^{2}=4\left(x-e_{1}\right)\left(x-e_{2}\right)\left(x-e_{3}\right)$ with $e_{1}<e_{2}<e_{3}$, we may calculate this period integral using the arithmetic-geometric mean. This is defined on two positive real arguments $x$ and $y$ by $M(x, y)=\lim _{n \rightarrow \infty} x_{n}=$ $\lim _{n \rightarrow \infty} y_{n}$, where $x_{0}=x, y_{0}=y, x_{n+1}=\left(x_{n}+y_{n}\right) / 2, y_{n+1}=\sqrt{x_{n} y_{n}}$. We find (Gauss):

(9) $\Omega=4 \int_{e_{3}}^{\infty} \frac{d x}{y}=\frac{2 \pi}{M\left(\sqrt{e_{3}-e_{1}}, \sqrt{e_{3}-e_{2}}\right)}=\frac{2 \pi}{M(2.22689 \ldots, 0.938503 \ldots)}$

$$
=4.151687983086933049884175683507286 \ldots \text {. }
$$

4. The $L$-Series. The $L$-series for $E$ over $\mathbf{Q}$ is given by an Euler product which converges in the right half-plane $\operatorname{Re}(s)>3 / 2$ :

$$
L(E, s)=\left(1+5077^{-s}\right)^{-1} \prod_{p \neq 5077}\left(1-a_{p} p^{-s}+p^{1-2 s}\right)^{-1}=\sum_{n=1}^{\infty} a_{n} n^{-s},
$$


where $a_{p}(p \neq 5077)$ equals $p+1-N_{p}$ with $N_{p}$ as in Section 2. We have

$$
\Lambda(s):=N^{s / 2}(2 \pi)^{-s} \Gamma(s) L(E, s)=\int_{0}^{\infty} f\left(\frac{i y}{\sqrt{N}}\right) y^{s-1} d y,
$$

where $N=5077$ and $f(\tau)=\sum_{n=1}^{\infty} a_{n} e^{2 \pi i n \tau}(\tau \in \mathbf{C}, \operatorname{Im}(\tau)>0)$. The Weil-Taniyama conjecture states that $f(\tau)$ is a cusp form of weight 2 on $\Gamma_{0}(N)$. We will assume that it is true. (This could be checked by a finite computation in the 422-dimensional space of cusp forms of this weight and level, but we have not carried it out; thus this note could more properly be described as a simultaneous numerical verification of the Birch-Swinnerton-Dyer and Weil-Taniyama conjectures.) Then $f(\tau)$ satisfies the functional equation $f(-1 / N \tau)=N \tau^{2} f(\tau)$ and the analytic continuation and functional equation of $L(E, s)$ follow:

$$
\Lambda(s)=\int_{1}^{\infty} f\left(\frac{i y}{\sqrt{N}}\right)\left(y^{s-1}-y^{1-s}\right) d y=-\Lambda(2-s) .
$$

In particular, the order of $L(E, s)$ at $s=1$ is odd and the $r$ th derivative ( $r \geqq 1$ odd) is given by

$$
\begin{aligned}
\Lambda^{(r)}(1) & =2 \int_{1}^{\infty} f\left(\frac{i y}{\sqrt{N}}\right)(\log y)^{r} d y \\
& =2 \sum_{n=1}^{\infty} a_{n} \int_{1}^{\infty} e^{-2 \pi n y / \sqrt{N}}(\log y)^{r} d y .
\end{aligned}
$$

If $\Lambda(s)$ vanishes to order $\geqslant r$ at $s=1$, then integrating (11) once by parts gives

$$
L^{(r)}(1)=\frac{2 \pi}{\sqrt{N}} \Lambda^{(r)}(1)=2 r ! \sum_{n=1}^{\infty} \frac{a_{n}}{n} G_{r}\left(\frac{2 \pi n}{\sqrt{N}}\right),
$$

where

$$
G_{r}(x)=\frac{1}{(r-1) !} \int_{1}^{\infty} e^{-x y}(\log y)^{r-1} \frac{d y}{y} \quad(r \geqq 1) .
$$

The series (12) is rapidly convergent, because $G_{r}(x) \sim x^{-r} e^{-x}$ as $x \rightarrow \infty$, so it can be used to compute $L^{(r)}(1)$ if we have a good algorithm to compute $G_{r}(x)$.

The function $G_{1}(x)$ is the familiar exponential integral $\int_{1}^{\infty} e^{-x y} d y / y$, which can be calculated for small $x(x<3)$ by the power series

$$
G_{1}(x)=\log \frac{1}{x}-\gamma+\sum_{n=1}^{\infty} \frac{(-1)^{n-1}}{n \cdot n !} x^{n} \quad(\gamma=\text { Euler's constant })
$$

and for large $x(x>2)$ by the continued fraction expansion

$$
G_{1}(x)=\frac{e^{-x}}{x+\frac{1}{1+\frac{1}{x+\frac{2}{1+\frac{2}{x+\frac{3}{1+\ldots}}}}}}
$$


Taking 250 terms of the series in $(12)$ gives $L^{\prime}(1) \approx 0$ to 13 decimal places. But this implies that $L^{\prime}(1)=0$ exactly, since the main result of [2] implies that $L^{\prime}(1)$ is a simple multiple of the height of some rational point on $E$ ("Heegner point") and, as we have seen, $E$ contains no rational points of very small nonzero height. Since $L(s)$ has odd order, we have ord ${ }_{s=1} L(s) \geqq 3$.

In general, the functions $G_{r}(x)$ satisfy $G_{0}(x)=e^{-x}, G_{r}^{\prime}(x)=-(1 / x) G_{r-1}(x)$, so

$$
G_{r}(x)=P_{r}\left(\log \frac{1}{x}\right)+\sum_{n=1}^{\infty} \frac{(-1)^{n-r}}{n^{r} n !} x^{n}
$$

for some polynomial $P_{r}$ of degree $r$. To determine $P_{r}$, we use the integral representation:

$$
G_{r}(x)=\frac{1}{2 \pi i} \int_{c-i \infty}^{c+i \infty} \frac{\Gamma(s)}{s^{r}} x^{-s} d s \quad \text { any } c>0 .
$$

(To prove (13), we observe that the right-hand side satisfies the same recursive differential equations as $G_{r}(x)$ and tends to zero as $x \rightarrow \infty$.) Shift the path of integration in (13) to the left; then the residue at $s=-n$ gives the term $(-1)^{n-r} x^{n} / n^{r} n$ ! and the residue at $s=0$ gives $P_{r}(\log 1 / x)$. Hence,

$$
P_{r}(t)=\sum_{m=0}^{r} \gamma_{r-m} \frac{t^{m}}{m !} \quad \text { where } \Gamma(1+s)=\sum_{n=0}^{\infty} \gamma_{n} s^{n}
$$

Since by Euler-Maclaurin

$$
\log \Gamma(1+s)=-\gamma s+\sum_{n=2}^{\infty} \frac{(-1)^{n}}{n} \zeta(n) s^{n},
$$

we find, for $r=3$, the expansion

$$
G_{3}(x)=\frac{1}{6}\left(\log \frac{1}{x}-\gamma\right)^{3}+\frac{\pi^{2}}{12}\left(\log \frac{1}{x}-\gamma\right)-\frac{\zeta(3)}{3}+\sum_{n=1}^{\infty} \frac{(-1)^{n-1} x^{n}}{n^{3} n !}
$$

which converges for all $x$. Using this we find the value

$$
\begin{aligned}
\lim _{s \rightarrow 1} \frac{L(E, s)}{(s-1)^{3}} & =2 \sum_{n=1}^{\infty} \frac{a_{n}}{n} G_{3}\left(\frac{2 \pi n}{\sqrt{5077}}\right) \\
& \approx 1.7318499001193006897919750851
\end{aligned}
$$

using the terms for $n \leqslant 600$ (the error made in breaking off the series here can be estimated using (12) and the formulas $G_{3}(x) \sim x^{-3} e^{-x}$ and $\left|a_{n}\right| \leqq d(n) \sqrt{n}$, where $d(n)$ is the number of divisors of $n$ ).

2.

The results of the computations described in this section are summarized in Table 
TABLE 2

Computation of $L^{\prime}(1)$ and $L^{\prime \prime \prime}(1)$

\begin{tabular}{rrrrcc}
\hline$n$ & $a_{n}$ & $G_{1}\left(\frac{2 \pi n}{\sqrt{N}}\right)$ & $G_{3}\left(\frac{2 \pi n}{\sqrt{N}}\right)$ & $2 \sum_{1}^{n} \frac{a_{m}}{m} G_{1}\left(\frac{2 \pi m}{\sqrt{N}}\right)$ & $2 \sum_{1}^{n} \frac{a_{m}}{m} G_{3}\left(\frac{2 \pi m}{\sqrt{N}}\right)$ \\
\hline 1 & 1 & 1.93741992 & 2.26675143 & 3.87483985 & 4.53350286 \\
2 & -2 & 1.32687953 & .98498602 & 1.22108079 & 2.56353082 \\
3 & -3 & 1.00056041 & .54955613 & -.78004003 & 1.46441856 \\
4 & 2 & .78875755 & .34359041 & .00871752 & 1.80800897 \\
5 & -4 & .63840821 & .22972608 & -1.01273562 & 1.44044725 \\
6 & 6 & .52596620 & .16064962 & .03919678 & 1.76174648 \\
7 & -4 & .43894007 & .11604939 & -.46244901 & 1.62911861 \\
8 & 0 & .36992797 & .08592813 & -.46244901 & 1.62911861 \\
9 & 6 & .31419941 & .06487957 & -.04351647 & 1.71562470 \\
10 & 8 & .26856035 & .04977090 & .38618010 & 1.79525814 \\
50 & -22 & .00231086 & .00005681 & -.00236637 & 1.73179489 \\
100 & 22 & .00001521 & .00000013 & .00001335 & 1.73185001 \\
250 & 48 & .00000000 & .00000000 & .00000000 & 1.73184990 \\
\hline
\end{tabular}

5. The Conjecture. The conjecture of Birch and Swinnerton-Dyer predicts that $\operatorname{ord}_{s=1} L(E, s)=\operatorname{rank}(E)=3$ and that

$$
\lim _{s \rightarrow 1} \frac{L(E, s)}{(s-1)^{3}}=\Omega \cdot R \cdot \operatorname{Card}(\mathrm{III})
$$

where III is the (conjecturally finite) Tate-Shafarevich group of $E$ over Q. Equations (8) and (9) give

$$
\Omega \cdot R=1.731849900119300689791975085060154 \ldots
$$

which agrees with the right-hand side of (14) within the accuracy of our computations in Section 3. This strongly suggests that the conjecture is true and that III $=(1)$. We have checked, via a 2-descent (cf. [1]), that the 2-primary component of III is trivial.

6. Acknowledgment. We would like to thank J.-F. Mestre for some useful suggestions.

Note added in proof. It has now been verified by J.-P. Serre and J.-F. Mestre that the curve $E$ satisfies the Weil-Taniyama conjecture (cf. beginning of Section 4), thus unconditionally justifying the calculations in this paper.

Department of Mathematics

Reed College

Portland, Oregon 97202

Department of Mathematics

Brown University

Providence, Rhode Island 02912

Max-Planck-Institut für Mathematik

Gottfried-Claren-Strasse 26

5300 Bonn 3, West Germany

and

Department of Mathematics

University of Maryland

College Park, Maryland 20742 
1. A. Brumer \& K. Kramer, “The rank of elliptic curves,” Duke Math. J., v. 44, 1977, pp. 715-743.

2. B. Gross \& D. Zagier, "Points de Heegner et dérivées de fonctions L," C. R. Acad. Sci. Paris, v. 297, 1983, pp. 85-87

3. Y. I. MANIN, “Cyclotomic fields and modular curves," Uspekhi Mat. Nauk, v. 26, 1971, pp. 7-71; English transl. in Russian Math. Surveys, v. 26, 1971, pp. 7-78.

4. B. Mazur \& H. P. F. Swinnerton-Dyer, "Arithmetic of Weil curves," Invent. Math., v. 25, 1974, pp. 1-61.

5. J. TATE, “The arithmetic of elliptic curves," Invent. Math. v. 23, 1974, pp. 179-206.

6. J. TATE, Letter to J.-P. Serre, Oct. 1, 1979.

7. H. G. Zimmer, "On the difference of the Weil height and the Néron-Tate height," Math. Z., v. 147, 1976, pp. 35-51. 